\title{
ARTICLE \\ Effects of hydrocortisone on autobiographical memory retrieval in patients with posttraumatic stress disorder and borderline personality disorder: the role of childhood trauma
}

\author{
Sophie Metz ${ }^{1}$, Juliane Fleischer ${ }^{1}$, Matti Gärnter ${ }^{1}$, Sabrina Golde ${ }^{1}$, Moritz Duesenberg ${ }^{1}$, Stefan Roepke ${ }^{1}$, Oliver T. Wolf ${ }^{2}$, \\ Christian Otte $^{1}$ and Katja Wingenfeld ${ }^{1}$
}

\begin{abstract}
In a previous study, we found that patients with posttraumatic stress disorder (PTSD) and borderline personality disorder (BPD) showed better autobiographical memory (AM) retrieval after hydrocortisone administration than after placebo administration. Here we investigate the neural correlates of AM retrieval after hydrocortisone administration in patients with PTSD or BPD. We recruited 78 female participants for this placebo-controlled crossover study: 40 healthy controls, 20 patients with PTSD, and 18 patients with BPD (all without medication). All participants received an oral placebo or $10 \mathrm{mg}$ hydrocortisone in a randomized order before performing an AM task. Neural activity was monitored during the task by functional magnetic resonance imaging. Neural activation did not differ between the three groups during AM retrieval, neither in the placebo condition nor after hydrocortisone intake. Multiple regression analysis revealed that Childhood Trauma Questionnaire scores correlated positively with hydrocortisone effects on activation in the anterior medial prefrontal cortex (amPFC), ventrolateral prefrontal cortex (vIPFC), posterior cingulate cortex (PCC), angular gyrus, and cerebellum. These results suggest that hydrocortisone-induced neural activation pattern during AM retrieval is related to childhood trauma. Previously described effects in the hippocampus, which were absent in the current study, might be related to PTSD caused by trauma in adulthood. The effects of hydrocortisone on brain activation and how these effects are influenced by childhood trauma, trauma in adulthood, and PTSD symptoms should be determined in future studies.
\end{abstract}

Neuropsychopharmacology (2019) 44:2038-2044; https://doi.org/10.1038/s41386-019-0459-8

\section{INTRODUCTION}

In a previous study, we found that hydrocortisone improved memory retrieval in patients with posttraumatic stress disorder (PTSD) and borderline personality disorder (BPD) [1, 2]. However, the neural underpinnings of enhanced memory retrieval after hydrocortisone administration in patients with PTSD and BPD have not been investigated in one study $[1,2]$. In the present study, we aimed to further characterize the neural correlates of these effects using functional magnetic resonance imaging (fMRI).

Cortisol enhances memory consolidation and impairs memory retrieval in healthy individuals [3-6]. Accordingly, stress and glucocorticoids affect autobiographical memory (AM) retrieval $[1,2,7]$. Williams and Broadbent developed an AM test (AMT) [8] to investigate $A M$ retrieval. In this test, participants recall events from their past in response to an emotional cue word. Using this AMT, Buss et al. [7] found reduced AM retrieval after hydrocortisone administration in healthy young men. Similar results $[1,9,10]$ have been described, as well as contrasting evidence that AM retrieval is not altered after hydrocortisone administration in healthy controls [2].

Glucocorticoids bind to glucocorticoid receptors (GRs) and mineralocorticoid receptors, which are expressed at high density in the hippocampus and prefrontal cortex (PFC) [11]. These brain areas play a crucial role in an AM retrieval-associated neural network that includes the ventromedial PFC, the dorsolateral PFC, the anterior cingulate cortex (ACC), the posterior cingulate cortex (PCC), the superior and middle temporal gyri, and limbic structures such as the hippocampus and parahippocampus [10, 12-15]. Previously, we investigated the neural underpinnings of AM retrieval after hydrocortisone administration in healthy participants, and found reduced activation in the anterior medial PFC (amPFC) [16].

AM retrieval is altered in several mental disorders [15, 17, 18]. As discussed, patients with PTSD and BPD have better AM retrieval after hydrocortisone administration than healthy controls do [1, 2], suggesting a normalization of AM performance. These results are compatible with enhanced GR sensitivity [19] and enhanced feedback sensitivity of the HPA (hypothalamic-pituitary-adrenal) axis in patients with PTSD [20]. Interestingly, some studies have shown enhanced basal and stimulated cortisol release and reduced feedback regulation of the HPA axis in patients with BPD [21]; comorbid depressive symptoms seem to strongly influence these alterations [22].

Hippocampus and PFC volumes are reduced in patients with BPD and PTSD. These brain regions have a high density of corticosteroid receptors $[19,21,23-26]$. In addition, the PFC is

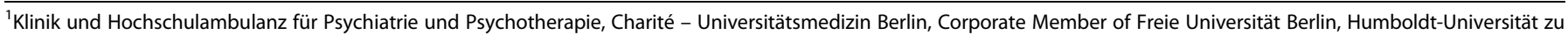

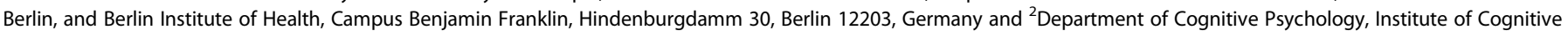
Neuroscience, Ruhr University Bochum, Bochum, Germany

Correspondence: Sophie Metz (sophie.metz@charite.de)

Received: 12 March 2019 Revised: 12 June 2019 Accepted: 28 June 2019

Published online: 6 July 2019 
hyporesponsive in patients with PTSD [24] and PFC metabolism is decreased in patients with BPD [21]. Alterations in glucocorticoid sensitivity $[27,28]$ and volumetric reductions in the PFC and hippocampus have also been described in individuals who experienced childhood trauma $[29,30]$. Therefore, childhood trauma may cause altered glucocorticoid sensitivity $[1,2]$ and volumetric reduction of the hippocampus and PFC [31, 32]. Early adversity might influence the response to glucocorticoids in adulthood, most likely via epigenetic mechanisms [33].

To our knowledge, only one study has investigated the effect of glucocorticoids on memory in patients with PTSD [34]. This study used positron emission tomography (PET) and showed that patients with PTSD have a hydrocortisone-associated increase in hippocampal $\left[{ }^{18} \mathrm{~F}\right]$ FDG uptake under resting state conditions. In addition, in a verbal declarative memory test following PET acquisition, PTSD patients did not show any hydrocortisoneassociated impairment in recall or retention. Furthermore, adrenocorticotropic hormone (ACTH) suppression was higher in PTSD patients after hydrocortisone administration, suggesting enhanced feedback sensitivity.

We hypothesized that effects of hydrocortisone on brain activity during AM retrieval would differ between healthy controls and patient groups, resulting in the following: (1) reduced activation of the hippocampus, PFC, ACC, PCC, and the superior and middle temporal gyri in healthy controls; and (2) an increased activation of these AM-related brain regions in patients with PTSD and BPD. As the majority of our PTSD and BPD patients reported early trauma, we additionally hypothesized that (3) childhood trauma correlates positively with increased activation of these AM-related brain regions after hydrocortisone administration.

\section{METHODS}

Participants

We recruited 40 healthy controls, 20 unmedicated patients with PTSD, and 18 unmedicated patients with BPD. The effects of hydrocortisone on AM retrieval in healthy individuals are not well described, so we decided to pre-analyze a subsample of our healthy control group. The paradigm used and data of these healthy controls were previously described [16]. Diagnoses were checked according to guidelines in the Structured Clinical Interview for Diagnostic and Statistical Manual of Mental Disorders (DSM-IV, SCID I \& II) [35]. Trained clinical psychologists (S.M., J.F.) conducted the interviews. The short version of the Borderline Symptom List (BSL-23) was used to measure severity of borderline symptoms [36] and the Posttraumatic Stress Diagnostic Scale (PDS-r) [37] was used to assess PTSD symptoms according to the DSM-IV. We assessed traumatic experiences in childhood with the Childhood Trauma Questionnaire (CTQ) [38], which consists of five sub-scores assessing emotional abuse, physical abuse, emotional neglect, physical neglect, and sexual abuse.

Individuals diagnosed with comorbid major depressive disorder (MDD), schizophrenia, schizoaffective disorder, bipolar disorder, and anorexia were excluded. Further exclusion criteria were Central Nervous System diseases, severe somatic diseases, metabolic or endocrine disorders, autoimmune diseases, current infections, pregnancy, and a body mass index (BMI) above 30 . Healthy controls were also excluded if they met the diagnostic criteria for any DSM-IV axis I or axis II disorder, had a history of psychiatric or psychotherapeutic treatment, or had taken any psychotropic medication. Any participants with fMRI contraindications (e.g., pacemaker or non-removable metals) were also excluded. Participants were all right-handed and native German speakers. Patients who fulfilled the diagnostic criteria for both BPD and PTSD were allocated to the BPD group.

Inpatients and outpatients were recruited at the Charite Universitätsmedizin Berlin, Klinik für Psychiatrie und Psychotherapie,
Campus Benjamin Franklin. Outpatients and healthy controls were recruited via advertisement and received financial remuneration (100 euros). Procedures were carried out with the participants' full understanding and written informed consent was obtained prior to participation. The study was approved by the local ethics committee.

\section{Procedure}

We used a placebo-controlled, double-blind crossover design, in which all participants performed the AMT during fMRI scanning after oral administration of either placebo or $10 \mathrm{mg}$ hydrocortisone in a randomized order.

Test sessions started at 3:30 pm. After hydrocortisone or placebo administration, participants rested for $45 \mathrm{~min}$. Participants then entered the scanner and were instructed to perform the AMT, while brain activity was recorded by fMRI. We repeated the measurements after a minimum 1-week interval. Throughout the afternoon, saliva samples were collected at five different time points (see Supplementary Materials).

\section{Autobiographical memory test}

The AMT was adapted from a test designed by Young et al. [15] and is based on the original AMT. There were two versions of the AMT used in the current study and each contained 25 words. Both versions were counterbalanced across conditions and stimuli were presented in a randomized order.

Similar to the method described by Young et al. [15], the participants were instructed to recall a specific event from their past in response to a particular cue word. Each cue word was presented for $15 \mathrm{~s}$. Standardized neutral, negative, and positive adjectives were selected as cue words based on previous literature $[1,2,7,39]$. These adjectives were used in pilot tests to determine whether they successfully induced AM retrieval. Fifty validated adjectives ( 25 adjectives for each version) were used in the final AMT with fMRI. Participants had to indicate whether they found a fitting memory by pressing a button on the response box. If they pressed a button, a fixation cross was presented for $10 \mathrm{~s}$ during which the participants were instructed to recall the AM. This was classified as remember condition when analyzing the data. After this recall period, participants rated the valence (neutral, negative, or positive), arousal (score from 1 [not arousing] to 4 [very arousing]), and recency (childhood, adolescence, or adulthood) of the retrieved memory. Answers were given by pressing the corresponding button on the response box. Participants were presented with a simple arithmetic problem as a distractor task [16]. Participants underwent three practice trials prior to fMRI scanning, in which the examiner explained the task, corrected mistakes, and answered all open questions. This was done directly after hydrocortisone or placebo intake.

\section{fMRI data acquisition}

Stimuli were presented using the software Presentation (Neurobehavioral Systems, Inc.) and the audio-visual stimulation technology VisuaStim Digital (Resonance Technology Company, Inc.). fMRI scans were obtained using a Siemens Magnetom TrioTim (3T) scanner with a 12-channel receiver coil array and an echoplanar imaging (EPI) pulse sequence $(3.0 \mathrm{~mm}$ slices acquired sagittally, repetition time $=2000 \mathrm{~ms}$, echo time $=30 \mathrm{~ms}$, flip angle $=70^{\circ}$, matrix $=64 \times 64$, field of view $=192 \mathrm{~mm}$, voxel size $=3 \times 3 \times 3 \mathrm{~mm}^{3}$ ). The actual number of acquired EPI images during the AMT strongly varied depending on the number of recalled memories with a maximum of 900 EPI images. Highresolution T1-weighted anatomical MRI scans were acquired for co-registration at the end of each MRI session.

\section{Data analysis}

Analysis of salivary cortisol concentration (to check hydrocortisone treatment) and behavioral data analysis are described in the Supplementary Materials section. 


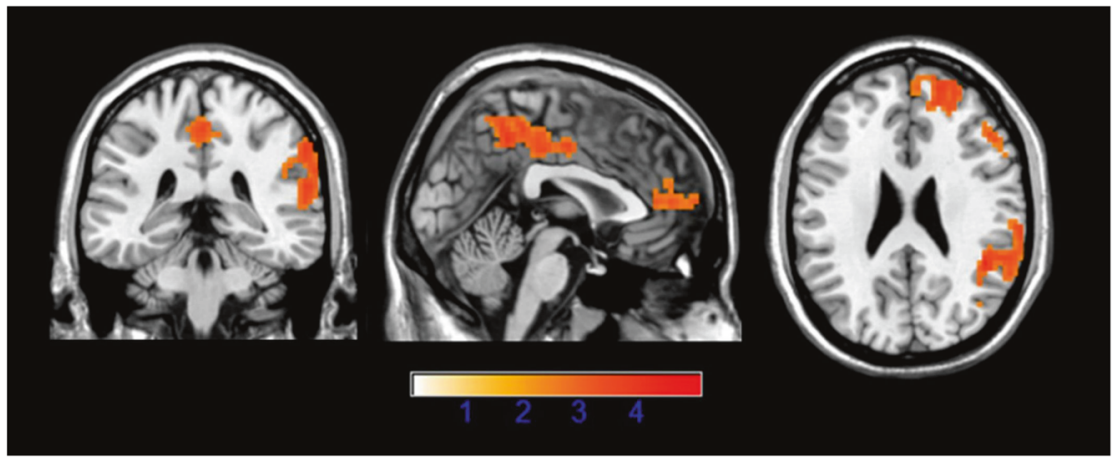

Fig. 1 Multiple regression of CTQ values on BOLD activity during the remember condition in the Autobiographical Memory Test (AMT) (contrast: hydrocortisone condition $>$ placebo condition). Clusters in amPFC, angular gyrus, PCC, and ventrolateral prefrontal cortex. For depiction, the threshold was set to $p<0.01$, uncorrected with an extent threshold of $k>200$ voxels. Values represent $Z$-values

\section{MRI analyses}

Image preprocessing and analyses were performed using SPM 12 (Welcome Trust Centre for Neuroimaging, UK; http://www.fil.ion. ucl.ac.uk/spm), under version of MathWorks MATLAB R2015a. Image preprocessing consisted of slice-timing, realignment to the mean image, and co-registration with anatomical images acquired at the end of the MRI session. Image data were normalized into MNI (Montreal Neurological Institute) space and smoothed using a six full width at half maximum kernel filter. For statistical analysis, a model for the different conditions convolved with a hemodynamic response function as explanatory variables and the six realignment parameters as additional regressors within the context of the general linear model on a voxel-by-voxel basis was implemented. A fixed-effect model at a single-subject level was used to create images of parameter estimates, which were then entered into a second-level random-effects analysis. For each subject, the following contrast images of parameter estimates were calculated: (1) Remember condition (placebo) vs. control condition (placebo) (Remember_placebo > Control_placebo); and (2) Remember condition (hydrocortisone) vs. remember condition (placebo) (Remember_hydro > Remember_placebo).

As a first step, we focused on brain activation during AM retrieval as described previously [15]. We examined brain activation during the contrast remember condition minus the control condition (simple arithmetic problem) in the placebo condition. This served to replicate the reported pattern of BOLD activation related to AM retrieval $[15,16]$. We carried out a onesample $t$-test to investigate differences across all groups and a one-way analysis of variance (ANOVA) to compare groups. Clusters were identified on a whole-brain level with family-wise error (FWE) cluster correction ( $p<0.05$, using a primary voxel-wise threshold of $p<0.001$, uncorrected, $k>10$ ). Second, we used images of the remember condition contrasting the hydrocortisone condition with the placebo condition. We conducted a one-sample $t$-test to investigate differences across all groups and a one-way ANOVA to compare the responses to hydrocortisone during the remember condition between the groups. Finally, we entered the abovedescribed contrasts into multiple regression analyses with CTQ scores to investigate the influence of childhood trauma on brain activation. Multiple regression using CTQ scores was carried out on the whole-brain level with FWE cluster correction $(p<0.05$, using a primary voxel-wise threshold of $p<0.001$, uncorrected, $k>10$ ).

\section{RESULTS}

Sample characteristics

Because of movement during scanning, we excluded data of three female healthy controls, one patient with PTSD, and one patient with BPD from the final analysis. Therefore, 37 healthy controls, 19 patients with PTSD, and 17 patients with BPD were included in the final analyses of fMRI data, behavioral data, and salivary cortisol concentrations. The groups did not differ in age, level of education, intake of contraceptives, menstrual cycle phase, smoking habits, and BMI (all $p>0.05$ ). As expected, the three groups differed in clinical measures (PDS-r, BSL-23, CTQ) (all $p<$ $0.001)$. Healthy controls scored lower in these questionnaires than patients with PTSD or BPD $(p<0.001)$ and patients with BPD had higher scores in the BSL-23 than patients with PTSD $(p<0.05)$. Patients with PTSD and BPD did not differ in CTQ and PDS-r scores (see Table 1). According to the SCID I interview, patients with PTSD reported comorbid bulimia nervosa $(n=2)$ and panic disorder $(n=3)$. Patients with BPD reported the following current axis I disorders: bulimia nervosa $(n=3), \operatorname{PTSD}(n=4)$, and social phobia $(n=2)$. Patients with BPD were previously diagnosed with the following: $\operatorname{MDD}(n=6)$, panic disorder $(n=1)$, anorexia nervosa $(n=1)$, bulimia nervosa $(n=1)$, agoraphobia $(n=1)$, adjustment disorder $(n=1)$, alcohol abuse $(n=3)$, alcohol dependency $(n=1)$, and substance abuse $(n=6)$. Patients with PTSD were previously diagnosed with the following: $\operatorname{MDD}(n=5)$, alcohol abuse $(n=2)$, alcohol dependency $(n=1)$, anorexia nervosa $(n=1)$, bulimia nervosa $(n=1)$, and substance abuse $(n=1)$. Furthermore, the majority of patients were outpatients $(n=16$ patients with BPD, $n=15$ patients with PTSD); only 2 patients with PTSD and 1 patient with BPD were inpatients. Two patients with PTSD were not seeking treatment but responded to the advertisement.

Salivary cortisol concentrations (to check hydrocortisone treatment) and behavioral data can be found in the Supplementary Information.

\section{FMRI analysis}

Autobiographical memory activation in the placebo condition. First, we investigated BOLD activity during the AMT in the placebo condition by contrasting the AM retrieval condition with the control condition across groups (Contrast: Remember_placebo > Control_placebo). Analyses revealed AM retrieval-related BOLD responses in the amPFC, superior frontal gyrus, angular gyrus, PCC, temporal cortex, and hippocampus across groups (see Table 2). These results fit to our prior analyses in healthy individuals [16]. These analyses showed whether this pattern of BOLD activation related to AM retrieval could be replicated in this larger sample. We investigated group differences with one-way ANOVA. The three groups did not differ in the contrast of the AM retrieval condition with the control condition.

Differences in hydrocortisone-induced changes in neural activity between groups. We compared the hydrocortisone condition and the placebo condition during AM retrieval (Contrast: Remember_hydro $>$ Remember_placebo) on the whole-brain level using a one- 
Table 1. Sample characteristics

\begin{tabular}{|c|c|c|c|c|c|}
\hline & & $\mathrm{HC}$ & PTSD & BPD & statistics \\
\hline Years of education, & & $12.12( \pm 1.32)$ & $11.37( \pm 1.83)$ & $11.76( \pm 1.52)$ & $p=0.25$ \\
\hline $\mathrm{BMI}^{\mathrm{b}}$ & & $21.94( \pm 2.51)$ & $22.90( \pm 3.47)$ & $22.52( \pm 4.23)$ & $p=0.71$ \\
\hline \multicolumn{6}{|l|}{ Menstrual cycle } \\
\hline $\mathrm{T} 1^{\mathrm{c}}$ & Luteal & $26 / 9$ & $9 / 8$ & $12 / 4$ & $p=0.25$ \\
\hline Intake of oral contraceptives ${ }^{e}$ & & $11 / 25$ & $2 / 17$ & $6 / 11$ & $p=0.18$ \\
\hline$B S L-23^{f}$ & & $0.11( \pm 0.10)$ & $1.08( \pm 0.71)$ & $1.76( \pm 0.76)$ & $\begin{array}{l}p<0.05 \\
\text { BPD }>\text { PTSD }>\text { HC }\end{array}$ \\
\hline PDS-r $r^{g}$ & & $0.28( \pm 0.88)$ & $29.50( \pm 13.27)$ & $24.87( \pm 14.43)$ & $\begin{array}{l}p<0.001 \\
\mathrm{PTSD}=\mathrm{BPD}>\mathrm{HC}\end{array}$ \\
\hline CTQ & & $30.59( \pm 5.21)$ & $63.50( \pm 24.42)$ & $65.71( \pm 22.36)$ & $\begin{array}{l}p<0.001 \\
\text { PTSD }=\text { BPD }>\text { HC }\end{array}$ \\
\hline
\end{tabular}

$B M I$ body mass index, BPD patients with borderline personality disorder, BSL-23 short version of the borderline symptom checklist, CTQ Childhood Trauma Questionnaire, $H C$ healthy controls, PDS posttraumatic stress diagnostic scale, PTSD patients with posttraumatic stress disorder, $T 1$ first measurement point, $T 2$ second measurement point. Missing data: ${ }^{\mathrm{a}} 1 \mathrm{HC},{ }^{\mathrm{b}} 2 \mathrm{HC},{ }^{\mathrm{C}} 2 \mathrm{HC}, 2$ Patients with PTSD, 1 Patient with BPD, ${ }^{\mathrm{d}} 3 \mathrm{HC}, 5$ patients with PTSD, 1 patient with BPD, ${ }^{\mathrm{e}} 1 \mathrm{HC}$, ${ }^{\mathrm{f}} 4$ patients with PTSD, 3 patients with BPD, ${ }_{5} \mathrm{HC}, 1$ patient with PTSD, 2 patients with BPD

Table 2. Autobiographical Memory Test (AMT). BOLD activity during the AMT in the placebo condition (contrast "recall vs. calculate")

\begin{tabular}{llll}
\hline Region & Side & Recall > calculate & Cluster size \\
\hline amPFC & $\mathrm{L}$ & $-125625(Z>8)$ & 1977 \\
PCC & $\mathrm{L}$ & $-6-5528(Z>8)$ & 1031 \\
Angular gyrus & $\mathrm{L}$ & $-48-7037(Z>8)$ & 1017 \\
Anterior temporal cortex & $\mathrm{R}$ & $60-7-17(Z: 6.11)$ & 130 \\
Tempoparietal junction & $\mathrm{R}$ & $54-6434(Z>8)$ & 119 \\
Hippocampus & $\mathrm{R}$ & $24-13-17(Z: 7.47)$ & 81 \\
& $\mathrm{~L}$ & $-21-19-17(Z: 6.79)$ & 99 \\
Superior temporal cortex & $\mathrm{R}$ & $48-371(Z: 6.01)$ & 55 \\
SFG & $\mathrm{R}$ & $184140(Z: 4.44)$ & 47 \\
\hline
\end{tabular}

amPFC anterior medial prefrontal cortex, $L$ left, $P C C$ posterior cingulate cortex, $R$ right, SFG superior frontal gyrus. Local maxima derived from whole-brain analyses with Family Wise Error (FWE) cluster correction $(p<$ 0.05 , using a primary voxel-wise threshold of $p<0.001$, uncorrected, $k>10$ ). The values in the table represent $z$-values with peak voxel coordinates in the MNI stereotactic space.

BOLD activity during the AMT in the placebo condition (contrast "recall vs. calculate")

sample $t$-test. We did not find an effect of condition. We also used one-way ANOVA to compare the response to hydrocortisone administration between groups using the same contrast (Contrast: Remember_hydro>Remember_placebo) on the whole-brain level. We did not find a main effect of group. Thus, neural activation patterns during AM retrieval did not differ between the three groups, neither in the placebo condition nor after hydrocortisone administration.

Multiple regression analysis using CTQ total scores revealed clusters on the whole-brain level of the contrast hydrocortisone condition minus placebo condition in the amPFC, ventrolateral PFC (vIPFC), PCC, angular gyrus, and cerebellum (see Table 3 and Fig. 1). Higher CTQ scores were associated with higher activation in the hydrocortisone condition compared with the placebo condition in these brain areas (see Fig. 2). No cluster of activation was revealed for the reversed contrast.
Table 3. Multiple regression of CTQ values on BOLD activity during the remember condition of the Autobiographical Memory Test (AMT) (contrast: hydrocortisone condition > placebo condition)

\begin{tabular}{|c|c|c|c|}
\hline Region & Side & $\begin{array}{l}\text { Hydrocortisone > } \\
\text { Placebo }\end{array}$ & Cluster size \\
\hline Angular gyrus & $\mathrm{R}$ & $42-5231$ (Z: 4.49) & 190 \\
\hline Cerebellum & $\mathrm{L}$ & $-18-82-29(Z: 4.86)$ & 59 \\
\hline amPFC & $\mathrm{R}$ & 185631 (Z: 4.24) & 49 \\
\hline PCC & $\mathrm{L}$ & $-6-4352(Z: 3.82)$ & 45 \\
\hline $\begin{array}{l}\text { Ventrolateral } \\
\text { prefrontal cortex }\end{array}$ & $\mathrm{R}$ & $5141-2(Z: 4.62)$ & 42 \\
\hline \multicolumn{4}{|c|}{$\begin{array}{l}\text { amPFC anterior medial prefrontal cortex, } L \text { left, } P C C \text { posterior cingulate } \\
\text { cortex, } R \text { right. Local maxima derived from whole-brain analyses with } \\
\text { Family Wise Error (FWE) cluster correction }(p<0.05 \text {, using a primary voxel- } \\
\text { wise threshold of } p<0.001 \text {, uncorrected, } k>10) \text {. The values in the table } \\
\text { represent } z \text {-values with peak voxel coordinates in the MNI } \\
\text { stereotactic space }\end{array}$} \\
\hline
\end{tabular}

\section{DISCUSSION}

We did not find differences in hydrocortisone-induced neural activation pattern during AM retrieval between healthy individuals and patients with PTSD and BPD. However, as expected, CTQ scores were associated with hydrocortisone effects during AM retrieval in the amPFC, vIPFC, PCC, angular gyrus, and cerebellum. These results suggest that changes in brain activity after hydrocortisone administration are related to childhood trauma across psychiatric diagnoses.

We replicated previously reported AM retrieval-associated activation in AM-related brain regions, including the amPFC and temporal brain regions. This activation pattern was seen in both patient groups and controls.

Effects of hydrocortisone on neural activity during autobiographic memory retrieval in PTSD and BPD patients

We found that neural activation during AM retrieval did not differ between PTSD or BPD patients and controls after administration of placebo or hydrocortisone. This in line with our behavioral results showing that group, condition, or an interaction of group and 

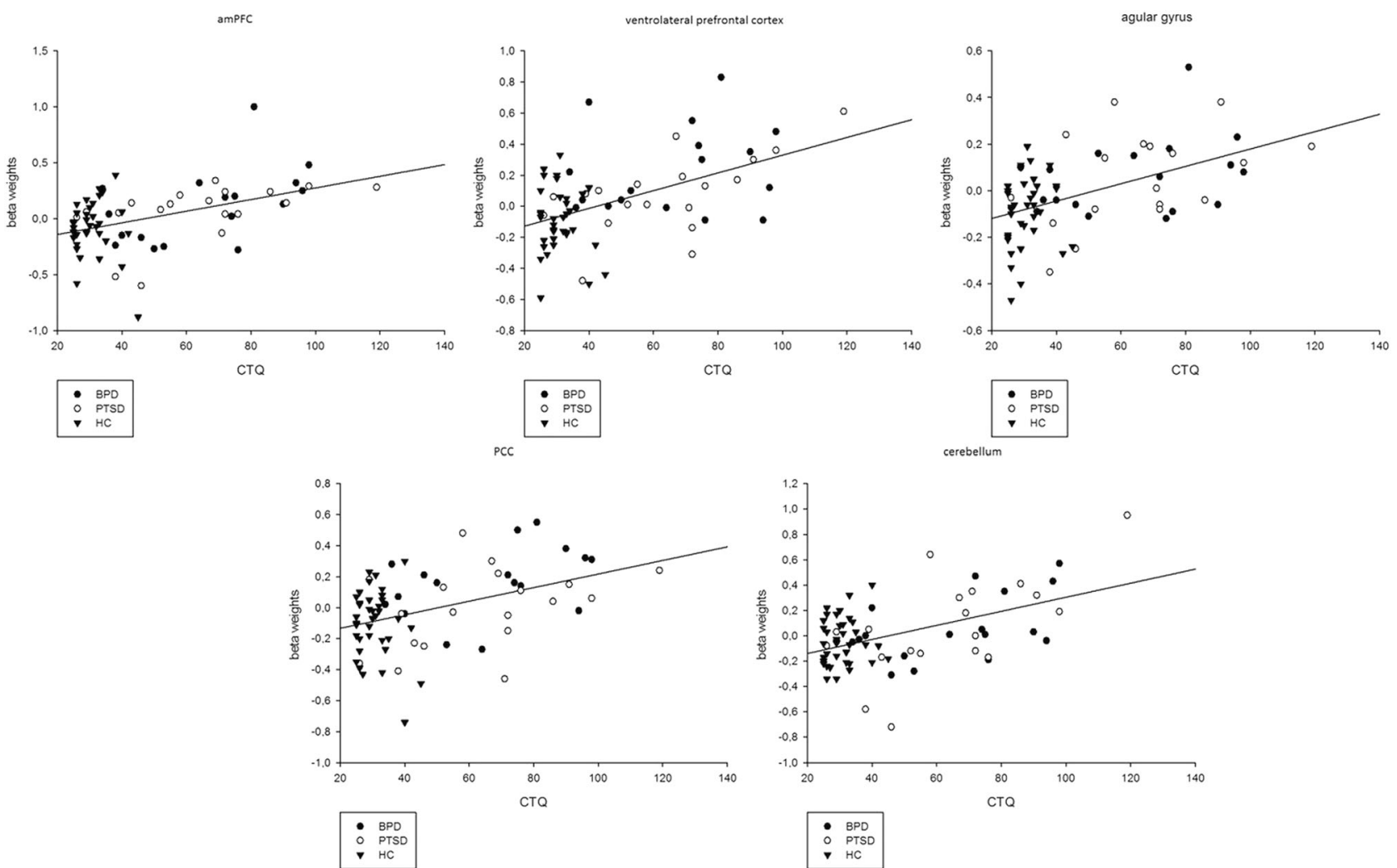

Fig. 2 Correlation of CTQ and beta weights of the contrast hydrocortisone minus placebo condition in amPFC, angular gyrus, PCC, ventrolateral prefrontal cortex, and cerebellum. amPFC anterior medial prefrontal cortex, BPD patients with borderline personality disorder, CTQ Childhood Trauma Questionnaire, HC healthy controls, PCC posterior cingulate cortex, PTSD patients with posttraumatic stress disorder

condition had no effect on the number of memories retrieved (see Supplementary Information). Thus, we did not see the expected decrease in brain activation (namely in the hippocampus) in response to hydrocortisone in the control group and increase in brain activation in response to hydrocortisone in patients with PTSD and BPD.

To date, only one study has investigated the effects of hydrocortisone on brain activity in PTSD [34]. Yehuda et al. [34] investigated Gulf War veterans using PET, and showed that hippocampal metabolic activity was higher after hydrocortisone administration than after placebo administration in patients with PTSD. However, these changes were seen in the resting state condition, not during a memory task, which might explain the differences to our results. We found no significant difference in brain activity during memory retrieval in patients and controls, regardless of hydrocortisone stimulation. In contrast to our hypotheses, we did not find difference in hippocampal activation. Former studies have shown that hydrocortisone impairs declarative memory retrieval of word pairs learned $24 \mathrm{~h}$ prior to cued recall. This was accompanied by a change in parahippocampal activation during PET in healthy controls [40]. This discrepancy between findings might be due to the different tasks that were used [16]; the declarative memory retrieval task showed differences in hippocampal activation whereas the AM retrieval task may affect the PFC. Interestingly, a meta-analysis showed the strongest activation in prefrontal areas during $A M$ retrieval compared with other memory tasks such as retrieval of recently learned stimuli $[41,42]$. These areas, which included the vmPFC and amPFC, are strongly involved in processing self-relevant information. In our previous study, activation was strongest in the amPFC during AM retrieval (placebo condition) [16]. In contrast, the hippocampus might play a less important role in AM retrieval compared with other episodic memory processes, and therefore is less activated during AM retrieval. This may explain why the hippocampus, contradictory to the assumption in our former publication [1, 2], seems to be less sensitive to cortisol effects during $\mathrm{AM}$ retrieval compared with other memory tasks, despite its high density of GR.

Yehuda et al. [34] investigated male war veterans while we examined women who predominantly reported childhood trauma. Participants in the study of Yehuda et al. [34] did not have different CTQ scores, which may have influenced the response to glucocorticoids (see next section). Moreover, PTSD symptoms (PDS-r scores) did not differ in severity between the patient groups in the current study, whereas the severity of BPD symptoms (BSL-scores) did differ. One might argue that the symptoms were not PTSD specific enough to reveal disorder-specific effects in PTSD patients. In sum, this study did not provide evidence for altered brain activation during AM retrieval or for altered brain function in response to hydrocortisone during AM retrieval in patients with PTSD or BPD. To which extent these results are transferable to other memory tasks needs to be investigated.

The role of childhood trauma in effects of hydrocortisone on neural activity during autobiographic memory retrieval CTQ scores correlated positively with hydrocortisone-induced activation of the amPFC, VIPFC, PCC, angular gyrus, and cerebellum during AM retrieval. Thus, self-reported childhood trauma seems to be positively associated with hydrocortisoneinduced activation of these brain areas.

Activation of these brain areas is related to AM retrieval [15, 42]. The amPFC is involved in self-referential processes [42], the vIPFC in retrieval, the cerebellum in executive processes, the parietal 
cortex in retrieval of spatial information, and the PCC in visuospatial processing [41].

We previously showed that hydrocortisone improved or normalized memory retrieval in patients with PTSD and BPD $[1,2]$. Of note, there was a high prevalence of childhood trauma in these patients. Enhanced GR sensitivity [19] and enhanced feedback sensitivity of the HPA axis was shown in patients with PTSD [20] and enhanced basal and stimulated cortisol release and reduced feedback regulation of the HPA axis in patients with BPD [21]. The importance of childhood trauma in altered corticosteroid receptor functioning has been discussed [1, 2, 43, 44]. Decreased hippocampal GR expression [45] has been described in individuals who experienced childhood trauma. Activation of hippocampal GRs decreases HPA axis activity, so an increase in pituitary ACTH, which reflects central activation of the HPA axis, in responses to stress in participants with childhood trauma is in line with these results [46]. However, reduced cortisol reactivity in response to stress $[47,48]$ has also been shown in participants with childhood trauma and in patients with BPD [49]. Abnormal stress responses might be linked to GR expression and resemble a dysregulation of the HPA axis with adverse health outcomes [47, 48]. Hydrocortisone may have normalized altered HPA axis activation and brain activation caused by altered GR functioning in our participants with childhood trauma. This is in line with observations in rodents. Long-term potentiation (LTP) is impaired in adult rodents with early-life stress experiences. Corticosterone treatment enhanced LTP in these animals, but impaired LTP in control animals with no early-life stress experiences [50]. Impaired LTP due to childhood trauma may increase after corticosteroid administration. This may explain behavioral memory results in PTSD and BPD patients [1, 2]. Potential epigenetic mechanisms and GR functioning needs to be further investigated in participants with childhood trauma.

The effects of hydrocortisone on hippocampal metabolism in patients with and without PTSD [51], and how these effects are related to different kinds of trauma should be investigated in future studies.

\section{Strengths and limitations}

We recruited 73 participants, including patients with PTSD and BPD, and healthy controls. All patients were unmedicated and had very few comorbid mental disorders. Studies have shown that HPA axis alterations are strongly related to comorbid PTSD and MDD in patients with BPD $[1,22]$, so we excluded all patients with comorbid current diagnosis of MDD.

There are several limitations to our study. The main limitation is the missing behavioral data on AM performance. Collection of behavioral data usually requires verbal or written statements by participants, which is challenging when monitoring brain activation by $\mathrm{fMRI}$ during the AMT; verbal assessment during scanning leads to movement artefacts and memory recall after scanning may be inaccurate. We included behavioral data on valence, arousal, and recency (see Table S1), but did not assess the specificity of rated memories during scanning. This makes it difficult to compare the current findings with our previous results $[1,2]$. However, the primary aim of the current study was to investigate the neural underpinnings of $A M$ and not to replicate our former findings $[1,2]$. The nature and design of the study required a substantial change to the task and specificity could not be coded without ambiguity. Therefore, we elucidated the neural underpinnings of $A M$ (which was the aim of this study) at the expense of not being able to collect behavioral data on AM specificity. In addition, the CTQ assesses childhood trauma retrospectively, so the ability to retrieve $\mathrm{AM}$ and memory biases may have influenced the results.

Our sample only included women; sex differences in the effects of glucocorticoids have been reported [52], so our results may not be transferable to men. Although individuals with comorbid current diagnosis of MDD were excluded, other comorbidities such as lifetime substance abuse or previous MDD may have influenced the results [53]. In addition, we did not collect information about previous treatment. As psychotropic medication [54] and psychotherapy [55] influence cortisol levels, this might have been relevant in the current study.

Although the sample size of our patient groups is lower than in our earlier behavioral studies [1, 2], the overall sample size included in the correlation was relatively large compared with other studies. However, the sample size may have been too low to detect certain effects between groups.

\section{CONCLUSION}

Severity of childhood trauma correlated positively with hydrocortisone-induced activation of the amPFC, vIPFC, PCC, angular gyrus, and cerebellum during AM retrieval. These results suggest that childhood trauma affects responsiveness to hydrocortisone independent of PTSD and BPD diagnosis. Brain activation was not altered during AM retrieval in patients with PTSD or BPD. Effects that were observed in the hippocampus in earlier studies may be explained by PTSD due to trauma in adulthood. How hydrocortisone-induced brain activation is affected by childhood trauma and trauma in adulthood should be investigated in future studies. These studies should collect behavioral data on memory specificity.

\section{FUNDING AND DISCLOSURE}

The study was supported by the Deutsche Forschungsgemeinschaft (DFG) [DFG-Grant WI 3396/2-3 to KW, OTW and CO]. The DFG were not involved in the study design; the collection, analysis, and interpretation of data; the writing of the report; or the decision to submit the paper for publication. The authors declare no competing interests.

\section{ADDITIONAL INFORMATION}

Supplementary Information accompanies this paper at (https://doi.org/10.1038/ s41386-019-0459-8).

Publisher's note: Springer Nature remains neutral with regard to jurisdictional claims in published maps and institutional affiliations.

\section{REFERENCES}

1. Wingenfeld K, Driessen M, Terfehr K, Schlosser N, Fernando SC, Otte C, et al. Effects of cortisol on memory in women with borderline personality disorder: role of co-morbid post-traumatic stress disorder and major depression. Psychol Med. 2013;43:495-505.

2. Wingenfeld K, Driessen M, Terfehr K, Schlosser N, Fernando SC, Otte C, et al. Cortisol has enhancing, rather than impairing effects on memory retrieval in PTSD. Psychoneuroendocrinology. 2012;37:1048-56.

3. Wolf O, Atsak P, De Quervain D, Roozendaal B, Wingenfeld K. Stress and memory: a selective review on recent developments in the understanding of stress hormone effects on memory and their clinical relevance. J Neuroendocrinol. 2016;28 https://doi.org/10.1111/jne.12353.

4. Roozendaal B. Stress and memory: opposing effects of glucocorticoids on memory consolidation and memory retrieval. Neurobiol Learn Mem. 2002;78:578-95.

5. Wolf OT. Stress and memory retrieval: mechanisms and consequences. Curr Opin Behav Sci. 2017;14:40-6.

6. Shields GS, Sazma MA, McCullough AM, Yonelinas AP. The effects of acute stress on episodic memory: a meta-analysis and integrative review. Psychol Bull. 2017;143:636.

7. Buss C, Wolf OT, Witt J, Hellhammer DH. Autobiographic memory impairment following acute cortisol administration. Psychoneuroendocrinology. 2004;29:1093-6.

8. Williams JM, Broadbent K. Autobiographical memory in suicide attempters. J Abnorm Psychol. 1986:95:144-9.

9. Schlosser N, Wolf OT, Fernando SC, Riedesel K, Otte C, Muhtz C, et al. Effects of acute cortisol administration on autobiographical memory in patients with major depression and healthy controls. Psychoneuroendocrinology. 2010;35:316-20. 
10. Young K, Drevets WC, Schulkin J, Erickson K. Dose-dependent effects of hydrocortisone infusion on autobiographical memory recall. Behav Neurosci. 2011; 125:735-41

11. Patel PD, Katz M, Karssen AM, Lyons DM. Stress-induced changes in corticosteroid receptor expression in primate hippocampus and prefrontal cortex. Psychoneuroendocrinology. 2008;33:360-7.

12. Young KD, Bellgowan PS, Bodurka J, Drevets WC. Behavioral and neurophysiological correlates of autobiographical memory deficits in patients with depression and individuals at high risk for depression. JAMA Psychiatry. 2013;70:698-708.

13. Young KD, Bellgowan PS, Bodurka J, Drevets WC. Neurophysiological correlates of autobiographical memory deficits in currently and formerly depressed subjects. Psychol Med. 2014;44:2951-63.

14. Young KD, Bellgowan PS, Bodurka J, Drevets WC. Functional neuroimaging correlates of autobiographical memory deficits in subjects at risk for depression. Brain Sci. 2015;5:144-64.

15. Young KD, Erickson K, Nugent AC, Fromm SJ, Mallinger AG, Furey ML, et al. Functional anatomy of autobiographical memory recall deficits in depression. Psychol Med. 2012;42:345-57.

16. Fleischer J, Metz S, Düsenberg M, Grimm S, Golde S, Roepke $S$, et al. Neural correlates of glucocorticoids effects on autobiographical memory retrieval in healthy women. Behav Brain Res. 2019;359:895-902.

17. Brown AD, Root JC, Romano TA, Chang LJ, Bryant RA, Hirst W. Overgeneralized autobiographical memory and future thinking in combat veterans with posttraumatic stress disorder. J Behav Ther Exp psychiatry. 2013;44:129-34.

18. Beran E, Richman MJ, Unoka Z. Autobiographical memory impairment in borderline personality disorder: a quantitative meta-analysis interpreted in terms of the CaR-FA-X model. J Pers Disord. 2018;32:1-14.

19. Szeszko PR, Lehrner A, Yehuda R. Glucocorticoids and hippocampal structure and function in PTSD. Harv Rev psychiatry. 2018;26:142-57.

20. Yehuda R. Sensitization of the hypothalamic-pituitary-adrenal axis in posttraumatic stress disordera. Ann New Y Acad Sci. 1997;821:57-75.

21. Wingenfeld K, Spitzer C, Rullkötter N, Löwe B. Borderline personality disorder: hypothalamus pituitary adrenal axis and findings from neuroimaging studies. Psychoneuroendocrinology. 2010a;35:154-70.

22. Wingenfeld K, Spitzer C, Rullkotter N, Löwe B. Borderline personality disorder: hypothalamus pituitary adrenal axis and findings from neuroimaging studies. Psychoneuroendocrinology. 2010b;35:154-70.

23. Aguilar-Ortiz S, Salgado-Pineda P, Marco-Pallarés J, Pascual JC, Vega D, Soler J, et al. Abnormalities in gray matter volume in patients with borderline personality disorder and their relation to lifetime depression: a VBM study. PLOS ONE. 2018;13:e0191946.

24. Shin LM, Rauch SL, Pitman RK. Amygdala, medial prefrontal cortex, and hippocampal function in PTSD. Ann New Y Acad Sci. 2006;1071:67-79.

25. Brunner R, Henze R, Parzer P, Kramer J, Feigl N, Lutz K, et al. Reduced prefrontal and orbitofrontal gray matter in female adolescents with borderline personality disorder: is it disorder specific? Neuroimage. 2010:49:114-20.

26. Wrocklage KM, Averill LA, Scott JC, Averill CL, Schweinsburg B, Trejo M, et al. Cortical thickness reduction in combat exposed US veterans with and without PTSD. Eur Neuropsychopharmacol. 2017;27:515-25.

27. Heim C, Nemeroff CB. Neurobiology of early life stress: clinical studies. Semin Clin Neuropsychiatry. 2002;7:147-59.

28. Heim C, Nemeroff CB. The role of childhood trauma in the neurobiology of mood and anxiety disorders: preclinical and clinical studies. Biol psychiatry. 2001;49:1023-39.

29. Hanson JL, Nacewicz BM, Sutterer MJ, Cayo AA, Schaefer SM, Rudolph KD, et al. Behavioral problems after early life stress: contributions of the hippocampus and amygdala. Biol Psychiatry. 2015;77:314-23.

30. Frodl T, Reinhold E, Koutsouleris N, Reiser M, Meisenzahl EM. Interaction of childhood stress with hippocampus and prefrontal cortex volume reduction in major depression. J Psychiatr Res. 2010;44:799-807.

31. Teicher $\mathrm{MH}$, Anderson CM, Polcari A. Childhood maltreatment is associated with reduced volume in the hippocampal subfields $C A 3$, dentate gyrus, and subiculum. Proc Natl Acad Sci USA. 2012;109:E563-E572.

32. van Harmelen A-L, van Tol M-J, van der Wee NJ, Veltman DJ, Aleman A, Spinhoven $\mathrm{P}$, et al. Reduced medial prefrontal cortex volume in adults reporting childhood emotional maltreatment. Biol Psychiatry. 2010;68:832-8.

33. Tyrka AR, Price LH, Marsit C, Walters OC, Carpenter LL. Childhood adversity and epigenetic modulation of the leukocyte glucocorticoid receptor: preliminary findings in healthy adults. PLoS ONE. 2012;7:e30148.
34. Yehuda R, Golier JA, Bierer LM, Mikhno A, Pratchett LC, Burton CL, et al. Hydrocortisone responsiveness in Gulf War veterans with PTSD: effects on ACTH, declarative memory hippocampal [18F] FDG uptake on PET. Psychiatry Res. 2010a;184:117-27.

35. Wittchen $\mathrm{H}$, Zaudig M, Fydrich T. SKID-I und SKID Strukturiertes klinisches interview für DSM-IV. Achse I und II. Göttingen: Hogrefe; 1995.

36. Bohus $M$, Kleindienst $N$, Limberger $M F$, Stieglitz R-D, Domsalla $M$, Chapman AL, et al. The short version of the Borderline Symptom List (BSL-23): development and initial data on psychometric properties. Psychopathology. 2009;42:32-9.

37. Foa, E. B. Posttraumatic stress diagnostic scale (PDS). Minneapolis, MN: National Computer Systems; 1995.

38. Bernstein D, Fink L. CTQ: Childhood Trauma Questionnaire: a retrospective selfreport. San Antonio, TX: Psychological Corp; 1998

39. Barnhofer T, Crane C, Spinhoven P, Williams JMG. Failures to retrieve specific memories in previously depressed individuals: random errors or content-related? Behav Res Ther. 2007:45:1859-69.

40. de Quervain DJ, Henke K, Aerni A, Treyer V, McGaugh JL, Berthold T, et al. Glucocorticoid-induced impairment of declarative memory retrieval is associated with reduced blood flow in the medial temporal lobe. Eur J Neurosci. 2003;17:1296-302.

41. Svoboda E, McKinnon MC, Levine B. The functional neuroanatomy of autobiographical memory: a meta-analysis. Neuropsychologia. 2006;44:2189-208.

42. Kim H. A dual-subsystem model of the brain's default network: self-referential processing, memory retrieval processes, and autobiographical memory retrieval. Neuroimage. 2012;61:966-77.

43. Fernando SC, Beblo T, Schlosser N, Terfehr K, Otte C, Löwe B, et al. Associations of childhood trauma with hypothalamic-pituitary-adrenal function in borderline personality disorder and major depression. Psychoneuroendocrinology. 2012;37:1659-68.

44. Bremner JD, Vythilingam M, Vermetten E, Adil J, Khan S, Nazeer A, et al. Cortisol response to a cognitive stress challenge in posttraumatic stress disorder (PTSD) related to childhood abuse. Psychoneuroendocrinology. 2003;28:733-50.

45. McGowan PO, Sasaki A, D'alessio AC, Dymov S, Labonté B, Szyf M, et al. Epigenetic regulation of the glucocorticoid receptor in human brain associates with childhood abuse. Nat Neurosci. 2009;12:342.

46. Heim C, Newport DJ, Wagner D, Wilcox MM, Miller AH, Nemeroff CB. The role of early adverse experience and adulthood stress in the prediction of neuroendocrine stress reactivity in women: a multiple regression analysis. Depress Anxiety. 2002b;15:117-25.

47. Lovallo WR, Farag NH, Sorocco KH, Cohoon AJ, Vincent AS. Lifetime adversity leads to blunted stress axis reactivity: studies from the Oklahoma Family Health Patterns Project. Biol psychiatry. 2012;71:344-9.

48. Lovallo WR. Early life adversity reduces stress reactivity and enhances impulsive behavior: Implications for health behaviors. Int J Psychophysiol. 2013;90:8-16.

49. Duesenberg M, Wolf OT, Metz S, Roepke S, Fleischer J, Elias V, et al. Psychophysiological stress response and memory in borderline personality disorder. Eur J Psychotraumatology. 2019;10:1568134.

50. Champagne DL, Bagot RC, van Hasselt F, Ramakers G, Meaney MJ, De Kloet ER, et al. Maternal care and hippocampal plasticity: evidence for experience-dependent structural plasticity, altered synaptic functioning, and differential responsiveness to glucocorticoids and stress. J Neurosci. 2008:28:6037-45.

51. Yehuda R, Golier JA, Bierer LM, Mikhno A, Pratchett LC, Burton CL, et al. Hydrocortisone responsiveness in Gulf War veterans with PTSD: effects on ACTH, declarative memory hippocampal [(18)F]FDG uptake on PET. Psychiatry Res. 2010b;184:117-27.

52. Merz CJ, Wolf OT. Sex differences in stress effects on emotional learning. J Neurosci Res. 2017;95:93-105.

53. Tyrka A, Parade S, Welch E, Ridout K, Price L, Marsit C, et al. Methylation of the leukocyte glucocorticoid receptor gene promoter in adults: associations with early adversity and depressive, anxiety and substance-use disorders. Transl Psychiatry. 2016;6:e848.

54. Manthey L, Leeds C, Giltay EJ, van Veen T, Vreeburg SA, Penninx BW, et al. Antidepressant use and salivary cortisol in depressive and anxiety disorders. Eur Neuropsychopharmacol. 2011;21:691-9.

55. Mommersteeg P, Keijsers GP, Heijnen CJ, Verbraak MJ, van Doornen LJ. Cortisol deviations in people with burnout before and after psychotherapy: a pilot study. Health Psychol. 2006;25:243. 